\title{
Effects of Sijunzi Decoction on Small Intestinal T Lymphocyte Subsets Differentiation in Reserpine Induced Spleen Deficiency Rats
}

\author{
${ }^{1,2}$ Nuowei Zhang, ${ }^{3}$ Shining Guo, ${ }^{4}$ Huanrong Li,,${ }^{1,2,5}$ Jiefeng Li, ${ }^{1,}{ }^{2}$ Xiaolong $\mathrm{Xu},{ }^{1,2}$ Changrong Wan, \\ ${ }^{4}$ Hong Zhao ${ }^{1,2}$ Faqiang Liu, ${ }^{4}$ Junlan Zan, ${ }^{2}$ Bin Wang and ${ }^{1,2}$ Jianqin Xu \\ ${ }^{1}$ TCVM Laboratory, CAU-BUA TCVM Teaching and Research Team, College of \\ Veterinary Medicine, China Agricultural University, 100193 Beijing, P.R. China \\ ${ }^{2}$ Key Laboratory of Development and Evaluation of the Chemical and \\ Herbal Drugs for Animal Use, Ministry of Agriculture, 100193 Beijing, P.R. China \\ ${ }^{3}$ College of Veterinary Medicine, South China Agricultural University, 510642 Guangzhou, China \\ ${ }^{4}$ Beijing Key Laboratory of TCVM, CAU-BUA TCVM Teaching and Research Team, \\ Beijing University of Agriculture, 102206 Beijing, P.R. China \\ ${ }^{5}$ Hebei Institute of Animal Science and Veterinary Medicine, 071000 Baoding, P.R. China
}

\begin{abstract}
Sijunzi decoction (SJZ), composed of Dangshen (Codonopsis pilosula Nannf.), Baizhu (Atractylodes macrocephala Koidz.), Fuling (Tuckahoe) and Gancao (Radix glycyrrhiza) is well known as a classical Chinese traditional and herbal medicamentum aiming directly at spleen deficiency in Traditional Chinese Medicine (TCM). The present research is to evaluate the regulatory effect of SJZ on small intestinal T lymphocyte subsets differentiation in reserpine-induced spleen deficiency rats. Reserpine induced spleen deficiency rats were orally administrated with SJZ $(0.2 \mathrm{~mL} / 200 \mathrm{~g}, 0.6 \mathrm{~mL} / 200 \mathrm{~g}$ and $1.0 \mathrm{~mL} / 200 \mathrm{~g}$, body weight $)$ once a day for a period of 5 days. Clinical symptoms including body weight were observed every day, the small intestine damage and recovery by SJZ were detected by electron microscope scanning, small intestine lymphocytes were separated for $\mathrm{T}$ lymphocyte subsets detection by flow cytometry and $\mathrm{T}$ lymphocyte related cytokine expression was studied using quantitative real time PCR. Results showed that proper dose of SJZ can significantly promote the recovery of body weight and small intestine damage as well as regulate the differentiation of $\mathrm{CD}^{+}$, $\mathrm{CD}^{+} \mathrm{CD} 4^{+} \mathrm{T}$ lymphocyte subsets and the expression of Type 1 cytokines (IL-2, IFN- $\gamma$ and IL10). Besides, a significant $\mathrm{CD}^{-} \mathrm{CD} 8^{-} \mathrm{T}$ lymphocyte subset was found in reserpine treated and high dose SJZ administrated rats. Data from this study proved that SJZ possess a significant promoting effect on the amelioration of spleen deficiency, $\mathrm{CD} 4^{+} \mathrm{T}$ lymphocyte and cytokines related with regulation of $\mathrm{T}$ lymphocyte differentiation would be key targets of SJZ on spleen deficiency.
\end{abstract}

Key words: Sijunzi decoction, T lymphocyte subsets, reserpine induced, spleen deficiency, small intestine, China

\section{INTRODUCTION}

In Traditional Chinese Medicine (TCM), spleen represents a macroscopic concept of digestion, absorption and nutrition metabolism (Yin et al., 2004). Spleen deficiency is a common disease from clinical syndrome differentiation based on Zang-Fu theory and generally refers to a series of pathological symptoms such as body weight loss, watery diarrhea, abdominal distention and depression (Hijikata et al., 2008; Cheng, 2005 ) which were caused by deficiency of spleen-Qi (Qi is the life force that flows through living beings and it is the energy that enables each organ system to do its job) (Batliner, 2004). Many reasons including maladjustment between work and rest, stress, anxiety and some chronic disease may result in spleen deficiency. Researches on spleen deficiency mainly consisted of two respects. One is establish new spleen deficiency models and another is spleen deficiency treatment. Up to now, lots of methods were developed to establish spleen deficiency models which mainly divide into three kinds, method of starvation and exhausted (Li, 1991) breaking the spleen-Qi with potent Chinese herbal drugs and injection of Qi-inhibiting chemicals (Xu et al., 2003, 2004; Li et al., 2009).

Corresponding Author: Jianqin Xu, TCVM Laboratory, CAU-BUA TCVM Teaching and Research Team, College of Veterinary Medicine, China Agricultural University, No. 2 West Yuanmingyuan Road, 100193 Beijing, P.R. China 
Reserpine is a drug that is thought to induce gastrointestinal damage via the depression of adrenergic activity with an increase in the cholinergic tone (Yamaguchi et al., 1978) and was defined as a Qiinhibiting chemical in TCM. Experimental use of reserpine as an inductor of Qi-inhibiting is widely accepted as a convenient model of spleen deficiency in laboratory (Zhao et al., 2010; Jia et al., 2006; Mi et al., 2005; Lin et al., 2010). Traditional Chinese medicine prescriptions are always the first choice for the treatment of spleen deficiency (Gao et al., 2009; Zhang et al., 2009; $\mathrm{Xu}$ et al., 1996), common used prescriptions for spleen deficiency including Liujunzi Tang, Yigongsan, Guipi Tang and so on. Most of them were derived from Sijunzi decoction (SJZ) which is composed by Dangshen (Codonopsis pilosula Nannf.), Baizhu (Atractylodes macrocephala Koidz.), Fuling (Tuckahoe) and Gancao (Radix glycyrrhiza) (Liu et al., 2005; Dai et al., 2006). It is a basic prescription for the cure of spleen deficiency and is also one of the most famous prescriptions recorded by Prescription of Peaceful Benevolent Dispensary (a famous prescription standard that exerted a very significant contribution to the development of TCM in history and also is the first prescription standard that published by the government of the People's Republic of China).

Many researches on the use of SJZ were conducted by TCM researchers in recent history. Xu A.H. firstly proved that SJZ can enhance the clearance rate of iv charcoal particles as well as bone marrow cells inhibited by cyclophosphamide in mice (Xu et al., 1993). Later researches demonstrated SJZ possess many other functions such as radio-protective activities (Hsu et al., 1996; Chen and $\mathrm{Fu}, 1996)$, inhibition of gastric cancer cell growth (Zhao et al., 2002), increase the ability of $\mathrm{T}$ lymphocyte transformation and the activity of superoxide dismutase and glutathione pemfidase (Cheng et al., 2009). Simultaneously, researches on the treatment of spleen deficiency by SJZ found that SJZ could significantly improved the disequilibrium of gastric electrical activity and the microelement in spleen deficiency cattle and goat models (Zheng et al., 2007) as well as improving xylose absorption in spleen deficiency rats (Gao et al., 2009). Previous study in the lab discovered SJZ also plays a role in regulating the gastrointestinal hormone secretion in spleen deficiency Beijing ducks (Dong et al., 2006; Zhang et al., 2006). However, up to now, few reports was found of the SJZ on gastrointestinal immune system and the mechanism of SJZ on reserpine induced spleen deficiency is still unclear.

In the present study, to assess the effects of SJZ on small intestinal $\mathrm{T}$ lymphocyte subsets differentiation in reserpine induced spleen deficiency rats and to further explore the mechanism of SJZ on reserpine induced spleen deficiency, SD rat models were prepared by intraperitoneally injected with reserpine, the ameliorate effects of small intestine damage by SJZ were evaluated according to the clinical situation, microstructure of small intestine, $C D$ molecules differentiation and cytokine expression in the models. The results proved that SJZ decoction possess a significant promoting effect on the amelioration of spleen deficiency, CD4 ${ }^{+} \mathrm{T}$ lymphocyte and cytokines related with regulating of $\mathrm{T}$ lymphocyte differentiation would be key targets of SJZ decoction on spleen deficiency.

\section{MATERIALS AND METHODS}

Chinese crude drugs: Codonopsis pilosula Nannf., Atractylodes macrocephala Koidz., Tuckahoe and Radix glycyrrhiza were bought from Beijing Tongrentang Pharmaceutical Co., Ltd. (Beijing, China) Reserpine was purchased from Bangmin Pharmaceutical Co., Ltd. (Guangdong, China), Goat anti-mouse monoclonal antiCD3-FITC, anti-CD4-APC and anti-CD8-PE antibodies were purchased from Becton Dickinson (Oxford, UK), Trizol and RevertAid First Strand cDNA Synthesis kit were obtained from Invitrogen (Carlsbad, CA, USA), Brilliant SYBR Green QPCR master mix was brought from Stratagene (La Jolla, California, USA). All other reagents used were of analytical grade.

Animals: Male SD rats weighing 200-220 g were purchased from Beijing Laboratory Animal Center (Beijing, China). The rats were kept in polypropylene cages in an air-conditioned room at $24 \pm 1^{\circ} \mathrm{C}$ with $12 \mathrm{~h}$ light cycle and fed pathogen-free food and water. All the procedures were performed in strict accordance with internationally accepted principles and the P.R. China legislation on the use and care of laboratory animals.

Preparation of S.JZ: SJZ, a standard yin-yangenrichment prescription of TCM composed by $60 \mathrm{~g}$ of Codonopsis pilosula Nannf., $60 \mathrm{~g}$ of Atractylodes macrocephala Koidz., $60 \mathrm{~g}$ of Tuckahoe and $30 \mathrm{~g}$ of Radix glycyrrhiza was extracted by water boiling method. Briefly, the dried prescription of herbs (210 g) was extracted twice with 10 fold volumes of boiling water $(2100 \mathrm{~mL}$ ) for $2 \mathrm{~h}$ each time. The result decoction was filtrated and concentrated to $1 \mathrm{~g} \mathrm{~mL}^{-1}$ and then stored at $4^{\circ} \mathrm{C}$ before administration. One of main components in the decoction was determined by high performance liquid chromatography as $141.30 \mu \mathrm{g} \mathrm{mL} \mathrm{mL}^{-1}$ of glycyrrhizin $\left(\mathrm{C}_{42} \mathrm{H}_{62} \mathrm{O}_{16}\right)$ and the yield of glycyrrhizin is $2.83 \%$ that is in accordance with the specifications of the Chinese veterinary pharmacopoeia. 
Table 1: Primer sequences for detection of cytokines

\begin{tabular}{lllr}
\hline Description & Accession No. & Primer sequence & Production size (bp) \\
IFN- $\gamma$ & NM_138880.2 & 5'-ACGCCGCGTCTTGGTTTTGC-3' & 175 \\
IL-2 & NM_053836.1 & 5'-ACCGTCCTTTTGCCAGTTCCTCC-3' & 164 \\
& & 5'-CTCGGAGCTCTGCAGCGTGT-3' & \\
IL-10 & NM_012854.2 & 5'-TCCACCACAGTTGCTGGCTCATC-3' & 183 \\
& & 5'-CCGAGAGCTGAGGGCTGCCT-3' & \\
IL-4 & NM_201270.1 & 5'-CCATGGTTCTCTGCCTGGGGC-3' & 150 \\
& & 5'-GGCTTCCAGGGTGCTTCGCAA-3' & 118 \\
& NM_031144.2 & 5'-GTGGACTCATTCACGGTGCAGC-3' & \\
\hline
\end{tabular}

Experimental model and treatment protocol: The spleen deficiency pattern in rat was induced by the intraperitoneal injection of reserpine. After 1 week acclimatization, the rat were randomly assigned to reserpine group (30 rats) and normal control group (6 rats). They were intraperitoneally injected with reserpine or saline $0.5 \mathrm{mg} \mathrm{kg}^{-1}$ body weight for 1 week. About 6 rats from each group were sacrificed on day 8 th. The rest of the rats form reserpine group was divided into four groups (6 rats each group): $\mathrm{SJZ}_{0.2}, \mathrm{SJZ}_{0.6}, \mathrm{SJZ}_{1.0}$ group and saline group. Rats form SJZ groups were orally treated with different dosages of SJZ decoction $(0.2,0.6$ and $1.0 \mathrm{~mL}$ of SJZ decoction per $200 \mathrm{~g}$ body weight, equivalent to Sijunzi crude drugs $11.5,34.5$ and $57.5 \mathrm{~g} \mathrm{~kg}^{-1}$ of body weight) for 5 days. Matched saline group were treated with corresponding volume of saline. All rats from above four groups were sacrificed on day 13th.

Observation on the villus of duodenum membrane: The specimens from duodenum (15-25 $\mathrm{mm}$ from the pylorus) were cut into blocks measuring $5 \times 3 \times 2 \mathrm{~mm}$ and immersed in $2.5 \%$ glutaraldehyde in $0.1 \mathrm{M}$ cacodylate buffer $(\mathrm{pH}=7.4)$ for $24 \mathrm{~h}$. The samples were dehydrated in a graded series of alcohol and acetone and transferred into amyl acetate. After critical point drying, the blocks were mounted on aluminium plates with the peritoneal surface exposed and sputter-coated with gold-paladium. The examination was carried out with a scanning electron microscope (S-3400N, Hitachi, Japan).

Determination of $T$ lymphocyte subsets in small intestine: Rats from each group were sacrificed on day 8th (reserpine group and control group) or day 13th (SJZ treated groups and Saline group) for $\mathrm{T}$ lymphocyte subsets determination. Single lymphocyte suspension at a concentration of $1 \times 10^{6}$ cells $/ 100 \mu \mathrm{L}$ was separated from ileum segment and stained with isotype control or triplestained with anti-CD3-FITC, anti-CD4-APC and anti-CD8$\mathrm{PE}$ monoclonal antibody. Cells stained with isotypematched monoclonal antibodies were used to determine the level of background fluorescence. The fluorescence intensities, two hundred thousand cells from each sample were measured using the FACSCalibur machine. $\mathrm{CD}^{+}$, $\mathrm{CD}^{+}, \mathrm{CD}^{+} \mathrm{CD}^{+}$and $\mathrm{CD} 4^{-} \mathrm{CD} 8^{-}$subset cells were counted within gated $\mathrm{CD} 3^{+} \mathrm{T}$ lymphocytes. Results were analyzed using FlowJo Analysis Software (Version 5.7.2, Tree Star, Ashland, Oregon, USA).

Determination of $\mathrm{IFN}-\boldsymbol{\gamma}, \mathrm{L}-2, \Pi \mathrm{L}-4$ and $\mathrm{IL}-10$ expression in small intestine: The pattern of cytokine mRNA expression in duodenum was determined by standard Reverse Transcription-Polymerase Chain Reaction (RTPCR). In brief, total mRNAs of small intestinal tissues of individual rats were extracted using Trizol. The cDNA was synthesized from $1 \mu \mathrm{g}$ total RNA using RevertAid First Strand cDNA Synthesis kit following their instructions of Invitrogen. Brilliant SYBR Green QPCR master mix was used for RT-PCR and PCR was run for 40 cycles containing $95^{\circ} \mathrm{C}$ for $1 \mathrm{~min}, 56^{\circ} \mathrm{C}$ for $1 \mathrm{~min}$ and $72^{\circ} \mathrm{C}$ for $1 \mathrm{~min}$. Expression levels were determined using the relative threshold cycle $\left(\mathrm{C}_{t}\right)$ method as described by the manufacturer (Stratagene). The sequences of the sense and antisense primers used for amplification are shown in Table 1.

Statistical analysis: Data were expressed as mean $\pm \mathrm{SD}$. Statistical analysis was performed using an independent sample t-test available in the SPSS Software (Version 12.0, Chicago, USA) where $\mathrm{p}<0.05$ was considered significant.

\section{RESULTS}

Changes of signs and body weights: Rat from reserpine group showed poor appetite, loose stools, lassitude, grouping and hypotrichotrophy since day $3 \mathrm{rd}$. This condition was relaxed to a certain extent in SJZ administrated group and saline group after reserpine injection was ceased. Rat from $\mathrm{SJZ}_{0.2}$ and $\mathrm{SJZ}_{0.6}$ group showed a better appetite compared with other groups after SJZ has been continuously administrated for 3 days. No significant difference of other clinical symptoms has been observed among SJZ groups and saline group. The 
effect of reserpine on the body weight is shown in Fig. 1. Compared with control group, rats injected with reserpine showed a significant body weight loss from days 5-7. SJZ groups and saline group trend to recover body weight after reserpine ceased to inject (day 7th) however, a significant difference was observed between SJZ treated groups and saline group since day 11 which demonstrated administration of SJZ could accelerate the tendency of body weights recovery. No significant differences were observed between the $\mathrm{SJZ}_{0.2}, \mathrm{SJZ}_{0.6}$ and $\mathrm{SJZ}_{1.0}$ groups.

Effect of SJZ on the internal structure of the small intestine: Electron microscope pictures were shown in Fig. 2, the structure of duodenum villus in control group is normal with well-arranged intestinal villus and proper interspace (Fig. 2A), individual villus presents a thick body as an oblonga cylinder (Fig. 2 $\mathrm{A}_{1}$ ). About 7 days after injection with reserpine, the integrity of the duodenum was destroyed, villus were shown random disposition, thin body (Fig. 2B) and incomplete construction with absence of massive of cellula columno

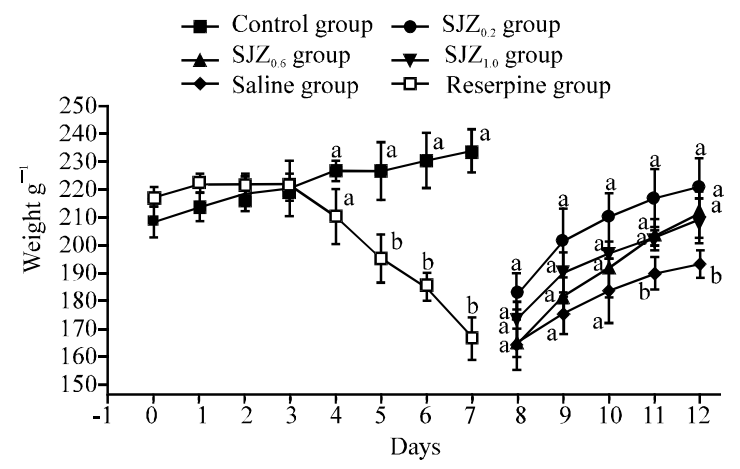

Fig. 1: Changes of the body weight in the rats from different groups. Letters $(a, b)$ denote significant differences $(p<0.05)$ epithelialis in villus top (Fig. $2 \mathrm{~B}_{1}$ ). Rats from $\mathrm{SJZ}_{0.2}$ and $\mathrm{SJZ}_{0.6}$ groups showed a relative complete villus top, less absence of cellula columnoepithelialis and many minor new-born cellula columnoepithelialis (arrow a, Fig. 2C, D) besides, a significant difference between these two groups is more fibrinous exudates can be observed in $\mathrm{SJZ}_{0.6}$ group. The villus of rats in $\mathrm{SJZ}_{1.0}$ group remaining seriously damaged, cellula columnoepithelialis of the villus exposed its basilar part (arrow b, Fig. 2 E) around the denudate lamina propria (arrow c, Fig. 2E). The structure of duodenum is unsharp in saline group, most of the villuses were covered by massive of disaggregated products and it is difficult to distinguish individual villus (Fig. 2F).

Changes on $\mathbf{T}$ lymphocyte subsets in small intestine: Presentation of $\mathrm{CD}^{+}, \mathrm{CD}^{+} \mathrm{CD} 4^{+}, \mathrm{CD} 3 \mathrm{CD} 8$, $\mathrm{CD}^{+} \mathrm{CD} 4^{-} \mathrm{CD} 8^{-} \mathrm{T}$ lymphocyte subsets in rat small intestine and the difference between different groups were shown in Fig. 3a-e. In normal control group, $\mathrm{CD}^{+}$, $\mathrm{CD}^{+} \mathrm{CD}^{+}, \mathrm{CD}^{+} \mathrm{CD}^{+} \mathrm{T}$ lymphocyte account for a proportion of $63.58,56.86$ and $20.21 \%$, separately (Fig. 3a-c).

A very small population of $\mathrm{CD} 4^{+} \mathrm{CD} 8^{+}$and $\mathrm{CD} 4^{-} \mathrm{CD} 8^{-}$ $\mathrm{T}$ lymphocytes, $<2 \%$ were detected. In reserpine treated group, the rats of $\mathrm{CD}^{+}, \mathrm{CD}^{+} \mathrm{CD} 4^{+}$and $\mathrm{CD}^{+} \mathrm{CD} 8^{+}$ $\mathrm{T}$ lymphocytes were significantly decreased to a lower proportion of $47.21,15.92$ and $3.18 \%$, separately (Fig. 3a-c). CD $4^{-} \mathrm{CD} 8^{-} \mathrm{T}$ lymphocytes, few was expressed in normal control group, increased significantly (from 1.98$4.56 \%$ ) and constructed a conspicuous $\mathrm{T}$ subpopulation (Fig. 3d). In SJZ or saline treated groups, $\mathrm{CD}^{+}$and $\mathrm{CD}^{+} \mathrm{CD}^{+} \mathrm{T}$ lymphocytes of $\mathrm{SJZ}_{0.2}$ group were significantly higher than that of $\mathrm{SJZ}_{0.6}, \mathrm{SJZ}_{1.0}$ and saline group. $\mathrm{CD}^{+} \mathrm{CD}^{+} \mathrm{T}$ lymphocytes of $\mathrm{SJZ}_{0.6}$ and $\mathrm{SJZ}_{1.0}$

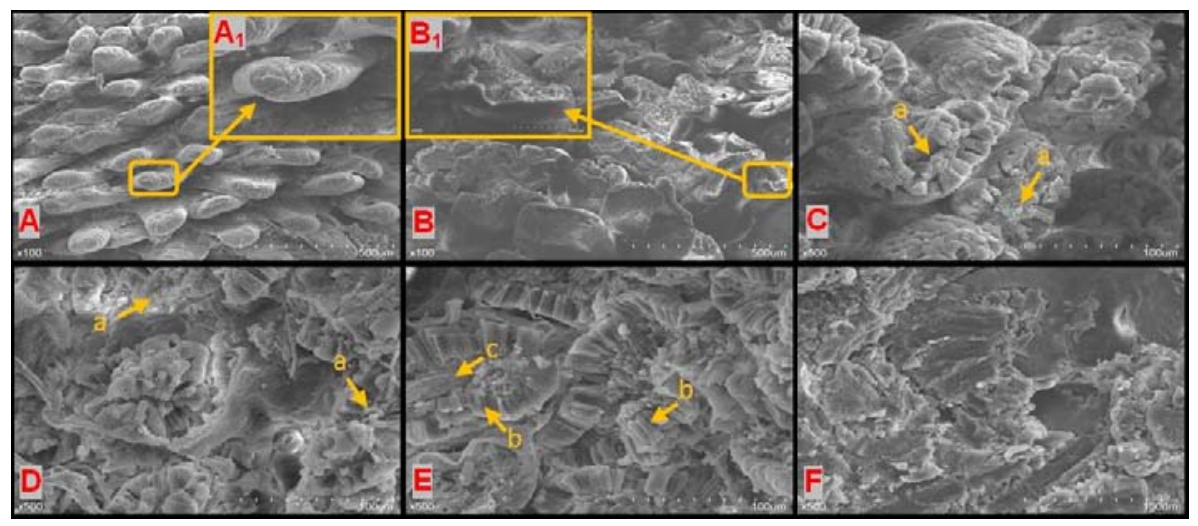

Fig. 2: Scanning electron microscope images of the small intestine. $A$ and $A_{1}$ ) Control group; $B$ and $B_{1}$ ) Reserpine group; C) $\mathrm{SJZ}_{0.2}$ group; D) $\mathrm{SJZ}_{0.6}$ group; E) $\mathrm{SJZ}_{1.0}$ group; F) Saline group where $\mathrm{A}_{1}, \mathrm{~B}_{1}, \mathrm{C}, \mathrm{D}, \mathrm{E}$ and $\mathrm{F}$ magnified 500 times, $A$ and $B$ magnified 100 times. Similar results were obtained in three rats from each group 

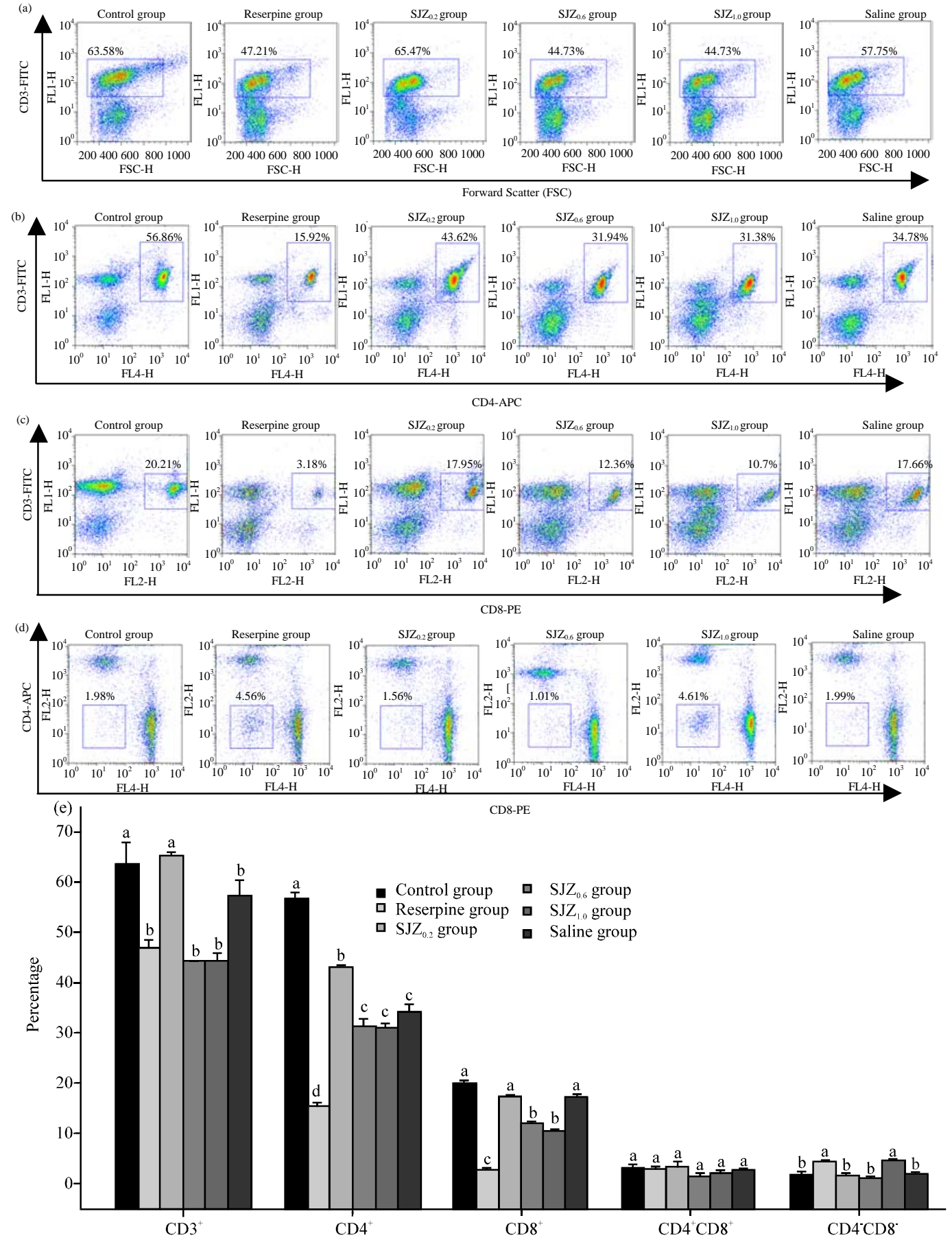

Fig. 3: Flow cytometric analysis of rat $\mathrm{T}$ cell subsections in small intestine. Cells in a-d were gated on $\mathrm{CD} 3^{+} \mathrm{T}$ cells, $\mathrm{CD}^{+} \mathrm{CD}^{+} \mathrm{T}$ cells, $\mathrm{CD}^{+} \mathrm{CD} 8^{+} \mathrm{T}$ cells and $\mathrm{CD}^{+} \mathrm{CD}^{-} \mathrm{CD}^{-} \mathrm{T}$ cells, separately. Similar results were obtained in three independent experiments. $\mathrm{e}$ is the $\mathrm{T}$ cell subsection changing between different groups. Data at the same time point with different letters $(\mathrm{a}-\mathrm{c})$ differed significantly $(\mathrm{p}<0.05)$

group were significantly lower than that of $\mathrm{SJZ}_{0.2}$ and saline group but no significant difference were observed between $\mathrm{SJZ}_{0.6}$ group and saline group. $\mathrm{CD} 4^{-} \mathrm{CD} 8^{-} \mathrm{T}$ lymphocytes were up regulated in
$\mathrm{SJZ}_{10}$ group and significantly higher than other SJZ or saline treated groups. Besides, no significant variation of $\mathrm{CD}^{+} \mathrm{CD}^{+} \mathrm{T}$ lymphocytes was detected in all groups. 


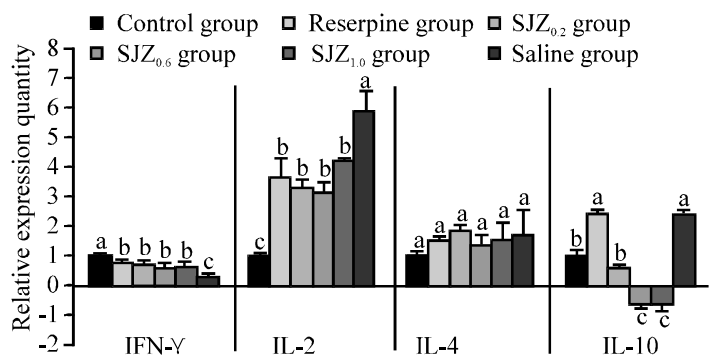

Fig. 4: Effect of SJZ on IL-2, IL-4, IL-10 and IFN- $\gamma$ expression in small intestine. Data at the same time point with different letters (a-d) differed significantly $(\mathrm{p}<0.05)$

Changes on $\Pi-2, \Pi-4, \Pi-10$ and $\mathbb{H N}-\gamma$ expression in small intestine: Compared with control group, expression of $\amalg-2$ and IL-10 were significantly enhanced while IFN- $\gamma$ was significantly depressed in reserpine treated group. This tendency kept on through the following treatment stage, especially IL-2 reached to a significant higher level and IFN- $\gamma$ reached to a significant lower level in saline group. However, in SJZ decoction administrated groups, the expression of $\mathrm{IL}-2$ and $\mathrm{IL}-10$ were significantly down regulated while IFN- $\gamma$ was significantly up-regulated compared with saline group. $\mathrm{SJZ}_{0.6}$ and $\mathrm{SJZ}_{1.0}$ groups showed a significant down regulation of IL-10 compared with all other groups. No significant differences were observed of IL-4 expression among all groups (Fig. 4).

\section{DISCUSSION}

Although, the TCM is widely utilized clinically as well as the Western medicine, differences do exist between those two kinds of medicine especially in the development process and evaluation of pharmacodynamics. The development process of Western medicine always begins with research on mechanism and ends with clinical verification. The influencing on biological effect of a certain target of the western medicine is usually regarded as a standard in evaluation of pharmacodynamics in western medicine. However, most of the new TCM, due to the compatibility of drugs, complex ingredients and multi-targets are not from laboratory but directly from the distilling of the daily clinical practice (Zhou et al., 2010) and hard to indentify its mechanism of pharmacologic action. To choose the most effective formula by clinical practice or clinical experiment of several different prescriptions is always the first step to develop a new TCM. Further investigation of the pharmacological properties and mechanism is always a posterior step. The history of SJZ is a typical development process of TCM. This classical formula was sieved from a great quantity of TCM specifically for curing stomach intestine diseases by long term clinical practice and its clinical effect has been proved by using in humanity and animals for hundreds of years history. With the development of science and technology, TCM scholars began focusing their attentions on the mechanism and new functions of SJZ in recent years. Up to now, research proved that SJZ could inhibit gastric cancer cell growth via induction the cell apoptosis and expression of $p 53$ and bcl-2 genes (Zhao et al., 2002), improve the immunomodulatory effects on Dgalactose-induced aging mice by increasing the ability of $\mathrm{T}$ lymphocyte transformation and increased the activity of superoxide dismutaseand glutathione pemfidase in serum (Cheng et al., 2009) as well as enhance immune function of organism by strengthening spleen and tonifying Qi (Zhang et al., 1999).

However, few researches with regard to the recovery effect of SJZ on spleen deficiency from the immunology point of view were carried out and the mechanism of SJZ on spleen deficiency is still unclear. It is well known that the epithelial cells of the gastrointestinal tract are continuously exposed to various toxic stimuli that may cause mucosal injury (Martinez et al., 2004). During injury, it is customarily the intestinal villi that get hurt at the very beginning, so the injury degree of intestinal villi is always regards as a standard to evaluate the damage. In the present research, electron microscope scanning on the small intestinal villi proved that the microstructural integrity of small intestine in rats of SJZ treated groups is obviously better than that in control group (mainly personification in the absence of intestinal villi epithelial cells) and among all SJZ treated group, $\mathrm{SJZ}_{0.2}$ and $\mathrm{SJZ}_{0.6}$ group have a more clear structure of intestine villi and less absent of epithelial cells. These results suggested that SJZ decoction at a proper dose can significantly enhance the recovery of small intestine damage in reserpineinduced spleen deficiency.

The cellular immune response is critical to the host defense system against infection by accelerating the clearance of pathogens and secreting many cytokines for the regulation of the immune response. $\mathrm{T}$ lymphocyte mediated immunity is usually regarded as a basic index when evaluating the cellular immune state in different organs. In this research, small intestinal $\mathrm{T}$ lymphocyte subsets differentiations were detected by flow cytometry. The expression of CD3 in reserpine treated group (47.21\%) and $\mathrm{SJZ}_{0.2}$ group $(65.47 \%)$ proved that $\mathrm{SJZ}_{0.2}$ can enhance CD3 molecule's expression in reserpine induced spleen deficiency. Most of the $\mathrm{T}$ lymphocytes express CD3 molecule and the expression of CD3 molecule always represent the differentiation state of $\mathrm{T}$ lymphocyte to a 
certain extent so, this result also indicated a role of SJZ in regulating $\mathrm{T}$ lymphocyte differentiation. Helper $\mathrm{T}$ lymphocyte (Th-cell, $\mathrm{CD}^{+} \mathrm{CD} 4^{+}$subset) and cytotoxic $\mathrm{T}$ lymphocyte (Tc-cell, $\mathrm{CD}^{+} \mathrm{CD}^{+}$subset) are two major $\mathrm{T}$ subsets defined by their selective surface expression of CD4 or CD8.

Th-cells are activate both humoral immune responses and cellular responses and Tc-cells show a major cytotoxic activity against cells infected with intracellular microbes and against tumor cells (Chaplin, 2010). Both effector Th-cells and Tc-cells can be subdivided into two cell subsets separately termed as Th1, Th2 and Tc1, Tc2 according to differences in their corresponding cytokine expression profiles. Thl and Tc1 produce mainly IL- 2 and IFN- $\gamma$ (type-1 cytokines) whereas Th2 and Tc2 produces IL-4 and IL-10 (type- 2 cytokines). Both Type 1 and 2 cytokines play an important role in regulating $\mathrm{T}$ lymphocyte differentiation. To evaluate $\mathrm{T}$ lymphocyte mediated immunity correctly, the differentiation state of two major $\mathrm{T}$ lymphocyte subsets and the expression of Type 1 and 2 cytokines were detected in this research. Result showed that SJZ can significantly influence the differentiation of Th cells but much less obvious to Tc cells. Simultaneously, the expressions of Type 1 cytokines and one of Type 2 cytokines (IL-10) were significantly regulated in SJZ group.

According to these result, we concluded that the effect of SJZ on $\mathrm{T}$ lymphocyte mediate immunity in reserpine induced spleen deficiency rats mainly depending on its regulation effect on Th-cell and Type 1 cytokines. With regard to the down regulation of $\mathrm{IL}-10$ in SJZ administrated groups, it is uncertain whether it was result from a direct effecting of SJZ. It is now known that IL10 not only has a key effect on the suppression of Th1-cell responses but also suppress the differentiation of Th2 cells (Hawrylowicz and O'Garra 2005). Down regulation of $\mathrm{IL}-10$ may indicate an enhancement of cell mediated immunity. Although, $\mathrm{IL}-10$ was originally isolated from Th2 cells, the expression of IL-10 is not specific to Th2 or Tc 2 cells but instead that it is a much more broadly expressed cytokine (Saraiva and O'Garra, 2010).

Except for $\mathrm{T}$ lymphocyte, many other cells of the of the innate immune system including dendritic cells, macrophages, mast cells, NK cells, eosinophils and neutrophils (Moore et al., 2001). Besides, IL-10 is an antiinflammatory cytokine with an important role in preventing inflammatory, the expression of IL-10 often reflect the level of inflammation. Accordingly, high-level expressions of $\mathrm{IL}-10$, the damage of the intestinal villus (results from scanning electron microscope images) in saline group and a low-level expression of $\mathrm{IL}-10$, the relative complete construction of intestinal villus made we believe that IL-10 may an important target, through which the inflammation degree could be significantly downregulated by SJZ. To sum up, the mechanism of SJZ on spleen deficiency has an important relation with small intestinal $\mathrm{T}$ lymphocyte differentiation activity. $\mathrm{CD}^{+} \mathrm{T}$ subset and related cytokines expression (IL2, IFN- $\gamma$ and IL-10) would be the main targets regulated by SJZ in spleen deficiency rats.

Apart from effector $\mathrm{T}$-cell subsets, accumulating evidence has demonstrated that Regulatory $\mathrm{T}$ cell (Treg) subsets also play an important role in the maintenance of immunologic self-tolerance and down-regulating various immune responses (Chen et al., 2004). The most classic Treg cell includes $\mathrm{CD}^{+} \mathrm{CD} 25^{+} \mathrm{FOXP}^{+} \mathrm{T}$ cell (Pillai et al., 2007), $\gamma \delta+T$ cell and NK cell (Von Bubnoff et al., 2010; $\mathrm{Lu}$ et al., 2007 ). $\mathrm{CD}^{-} \mathrm{CD}^{-} \mathrm{T}$ lymphocyte is a novel subset with regulating effect on eliminating the undesired effector clone via Fas/FasL mediated apoptosis (Fisher et al., 2005). Matured CD4 ${ }^{-} \mathrm{CD} 8^{-}$Treg cells are a subset of $\mathrm{T}$ lymphocytes that present less in the periphery (1-3\% of small intestinal $\mathrm{T}$ lymphocytes in this research) and educe depressant effect on effector $\mathrm{T}$ lymphocyte. Recent research found that $\mathrm{CD} 4^{-} \mathrm{CD} 8^{-} \mathrm{T}$ lymphocyte will express significantly in parasitic infection, heterogenic transplantation and some other immunological diseases (Nagib et al., 2007; Ling et al., 2007; Zhang et al., 2000; Priatel et al., 2011). However, there is no information on the occurrence of these cells in intestinal damage and recovery.

In this research, researchers find that $\mathrm{CD} 4^{-} \mathrm{CD} 8^{-} \mathrm{T}$ lymphocyte subset participated in intestine damage of spleen deficiency rats and SJZ exerted an effect on the expression of this subset. Results demonstrated that the influence SJZ on cell immunity is not only limited to major $\mathrm{T}$ lymphocyte subsets but also on smaller subsets with specific regulating function just like $\mathrm{CD} 4^{-} \mathrm{CD} 8^{-}$which may play a significant role in spleen deficiency rats. Beside, taking into account the damage and recovery situation of the small intestine in reserpine treated group and $\mathrm{SJZ}_{1.0}$ group detected by electron microscope scanning, researchers prefer to believe that high expression of $\mathrm{CD}^{-} \mathrm{CD} 8^{-} \mathrm{T}$ subset in reserpine treated group $(4.56 \%)$ and $\mathrm{SJZ}_{1.0}$ group $(4.61 \%)$ may imply this subset exerted an adverse regulating effect on $\mathrm{T}$ lymphocyte mediated immune response and is harmful for the amelioration of reserpine induced spleen deficiency. Chinese medical philosophy is characterized by its emphasis on the restoration and maintenance of balance (Wang et al., 2007). 


\section{CONCLUSION}

This study shows that the effect of SJZ on spleen deficiency rats including the amelioration of small intestine damage, recovery of $\mathrm{T}$ lymphocyte differentiation and cytokines expression proved SJZ is an effective TCM prescription and the pharmacologic mechanism of SJZ was coincidence with the theory of Chinese medical philosophy. In conclusion, SJZ exerts regulating effects on the recovery process of reserpine induced spleen deficiency mainly by regulating $\mathrm{CD}^{+} \mathrm{T}$ subset and cytokines related with $\mathrm{T}$ lymphocyte differentiation. $\mathrm{CD}^{-}{ }^{-} \mathrm{CD} 8^{-} \mathrm{T}$ subset participated in the reserpine induced spleen deficiency and the amelioration process regulated by SJZ but this subset may act as a suppressor on the amelioration of spleen deficiency.

\section{ACKNOWLEDGEMENTS}

This research was supported by grants from National Twelve Five Technological Supported Plan of China (No.: 2011BAD34B01), PHR (IHLB), Research Fund for the Doctoral Program of Higher Education of China and Public Service Sectors Agriculture Research Projects (No.: 201003060-9/10). Researchers are thankful for the help from the members of CAU-BUA TCVM teaching and research team.

\section{REFERENCES}

Batliner, A., 2004. Spleen Qi Deficiency A Nutritional Perspective. Nutrition Professionals Quarterly, New Jersey, USA.

Chaplin D.D., 2010. Overview of the immune response. J. Allergy Clin. Immunol., 125: s3-s23.

Chen, G.Z. and D. Fu, 1996. Effects of jiawei sijunzi tang decoction on migrating myoelectric complex in 8.0Gy irradiated rats. World J. Gastroenterol., 2: 197-199.

Chen, W.H., M.S. Ford, K.J. Young and L. Zhang, 2004. The role and mechanisms of double negative regulatory $\mathrm{T}$ cells in the suppression of immune respose. Cell. Mol. Immunol., 1: 328-335.

Cheng, W., 2005. Shanghan Lun zhujie. 2nd Edn., The People's Medical Publishing House, Beijing.

Cheng, W.L., X.Y. Wang, Z.Y. Jiang, J.Q. Pan, J. Dong, S.S. Kuang and Z.L. Rao, 2009. The immunomodulatory effects of sijunzi decoction and its disassembled prescription on D-galactoseinduced aging mice. Zhong Yao Cai., 32: 1425-1429.

Dai, X., J. Li, S. Chen and R. Jiang, 2006. Efect of Si-jun-zi decoction on lymphocyte flunction in spleen asthenia rat. Chin. J. Mod. Med., 16: 1288-1289.
Dong, H., F. Liu, X. Mu, T. Zhang and J. Xu, 2006. Effect of Sijunzi decoction on the expression of mRNA of VIP and SS of Beijing Duck with spleen deficiency. Acta Veterinaria et Zootechnica Sin, 37: 1042-1046.

Fisher, K., S. Voelkl, J. Heymann, G.K. Przybylski and $\mathrm{K}$. Mondal et al., 2005. Isolation and characterization of human antigen-specific TCRab+CD4-CD8- doublenegative regulatory T cells. Blood, 105: 2828-2835.

Gao, X.L., W.F. Guo, R.L. Li and W.W. Chen, 2009. Effects of sijunzi decoction on urine's xylose excretion rate and ATP in mucosa of spleen deficiency rats. Zhong Yao Cai., 32: 1242-1245.

Hawrylowicz, C.M. and A. O'Garra, 2005. Potential role of interleukin-10-secreting regulatory $\mathrm{T}$ cells in allergy and asthma. Nat. Rev. Immunol., 5: 271-283.

Hijikata, Y., N. Makiura, T. Kano, K. Higasa, M. Shimizu, K. Kawata and T. Mine, 2008. Kampo medicine, based on traditional medicine theory, in treating uncured glossodynia: Efficacy in five clinical cases. Am. J. Chin. Med., 36: 835-847.

Hsu, H.Y., J.J. Yang, S.L. Lian, Y.H. Ho and C.C. Lin, 1996. Recovery of the hematopoietic system by Si-Jun$\mathrm{Zi}$-Tang in whole body irradiated mice. J. Ethnopharmacol., 54: 69-75.

Jia, H., N. Zhao, W. Zhan, Z. Shan and Z. Zhang et al., 2006. Effects of lizhong wan and sijunzi tang on active movement of rats with spleen deficiency induced by reserpine. Chin. J. Inf. Traditional Chin. Med., 13: 34-35.

Li, Y.K., 1991.. Experimental Methodology of Traditional Chinese Medicine Pharmacology. Shanghai Science and Technology Publishing House, Shanghai.

Li, Z., G. Xiao, Z. Xu, G. Duan and X. Yang, 2009. Action of xiangshaliujun granule on gastrointestinal peristalsis in experimental rats and patients with spleen deficiency syndrome. World J. Gastroenterol., 17: $512-515$.

Lin, C.Q., Y.L. Chen and R.L. Li, 2010. Effect and mechanism of reserpine for changing salivary protein secretion in pi-deficient rats. Zhongguo Zhong $\mathrm{Xi} \mathrm{Yi}$ Jie He Za Zhi, 30: 509-512.

Ling, E., G. Shubinsky and J. Press, 2007. Increased proportion of CD3+CD4-CD8- double-negative $\mathrm{T}$ cells in peripheral blood of children with Behcet's disease. Autoimmun. Rev., 6: 237-240.

Liu, L., L. Han, D. Wong, P. Yue and W. Ha et al., 2005. Effects of Si-Jun- $\mathrm{Zi}$ decoction polysaccharides on cell migration and gene expression in wounded rat intestinal epithelial cells. Br. J. Nutr., 593: 21-29.

Lu, L., K. Ikizawa, D. Hu, M.B. Werneck, K.W. Wucherpfennig and H. Cantor, 2007. Regulation of Activated CD4+ T Cells by NK Cells via the Qa-1-NKG2A Inhibitory Pathway. Immunity, 26: 593-604. 
Martinez, G., G. Cantarella, V.M. Cutuli, C. Loreto and A. Prato et al., 2004. Effect of adrenomedullin on cMet receptor expression after reserpine-induced gastric damage in the rat. Eur. J. Pharmacol, 488: 219-224.

Mi, N., Q.Y. Chen, M.Y. Wu and W.M. Sun, 2005. Effect of radix astragali in buzhong yiqi decoction on immunoregulation in spleen-deficiency mice. World Chin. J. Digestol., 13: 963-966.

Moore, K.W., M.R. de Waal, R.L. Coffman and A. O'Garra, 2001. Interleukin-10 and the interleukin 10 receptor. Annu. Rev. Immunol., 19: 683-765.

Nagib, P.R.A., W.O. Dutra, E. Chiari and C.R.S. Machado, 2007. Trypanosoma cruzi: Populations bearing opposite virulence induce differential expansion of circulating CD3+CD4-CD8- T cells and cytokine serum levels in young and adult rats. Exp. Parasitol., 116: 366-374.

Pillai, V., S.B. Ortega, C.K. Wang and N.J. Karandikar, 2007. Transient regulatory T-cells: A state attained by all activated human T-cells. Clin. Immunol., 123: 18-29.

Priatel, J., O. Utting and H. Teh, 2011. TCR/Self-antigen interactions drive double-negative $\mathrm{t}$ cell peripheral expansion and differentiation into suppressor cells. J. Immunol., 167: 6188-6194.

Saraiva, M. and A. O'Garra, 2010. The regulation of IL-10 production by immune cells. Nat. Rev. Immunol., 10: $170-181$.

Von Bubnoff, D., E. Andres, F. Hentges, T. Bieber, T. Michel and J. Zimmer, 2010. Natural killer cells in atopic and autoimmune diseases of the skin. J. Allergy Clin. Immunol., 125: 60-68.

Wang, G., B. Mao, Z.Y. Xiong, T. Fan and X.D. Chen et al., 2007. The quality of reporting of randomized controlled trials of traditional Chinese medicine: A survey of 13 randomly selected journals from mainland China. Clin. Ther., 29: 1456-1467.

Xu, A., Y. Gong, W. Gu and X. Wang, 1993. Comparison of the effect of sijunzi decoction, siwu decoction and bazhen decoction on immune function in mice. Chin. J. Chin. Mater. Med., 18: 240-242.

Xu, G., J. Zhang and P. Yian, 1996. Changes of plasma cAMP and cGMP level in patients with spleen-qi deficiency in chronic atrophic gastritis. World J. Gastroenterol., 2: 226-227.

Xu, Q., J. Wang, R. Wang, X. Liu and Z. Chen et al., 2004. Effect of Buzhong Yiqi decoction on gastrin receptor in gastric parietal cells and its associated mechanisms of invigorating spleen in spleen-deficiency rat. Chin. J. Rehabil., 8: 2986-2987.
Xu, Q., Z. Chen, R. Wang, J. Wang and X. Liu, 2003. Effect of Buzhong Yiqi Tang on the gastrin receptors in the gastric parietal cells in rat with def iciency of the spleen. Pharmacol. Clin. Chin. Mater. Med., 19: 3-5.

Yamaguchi, I., J. Hiroi, H. Fuke and S. Kumada, 1978. Mechanism of gastric secretagogue effect of reserpine in rat. J. Pharmacol. Exp. Ther., 205: 710-717.

Yin, G.Y., W.N. Zhang, X.J. Shen, X.F. He and Y. Chen, 2004. Study on the pathological basis of classification of spleen deficiency in chronic gastritis. Chin. Med. J., 117: 1246-1252.

Zhang, M., Z. Zhang, T. Xia, Z. Wang, K. Sun, C. Xu and W. Yu, 1999. Effect of sijunzi decocition on serum soluble intercellular adhesive molecule-1, interleukin15 and monocyte antibody-dependent cell-mediated cytotoxicity in patients of spleen deficiency. Chin. J. Integr. Traditional Western Med., 19: 270-272.

Zhang, Y., H. Wang, X. Yang, S. Zhu, C. Bi and Y. Li, 2009. Affection of the chinese herbal drug decoction of yishi on the spleen-qi deficiency models reconstruction of reattached retina. Int. J. Ophthalmol., 9: 2295-2297.

Zhang, Y.D., H.Q. Duan, H. Dong, X. Mu, K. Fan, J.Q. Xu and F.H. Liu, 2006. Effects of sijunzi decoction on expression of CCK and VIP mRNA in peking duck duodenum and jejunum. Acta Veterinaria et Zootechnica Sin., 37: 809-813.

Zhang, Z.X., L. Yang, K.J. Young, B. DuTemple and L. Zhang, 2000. Identification of a previously unknown antigen-specific regulatory $\mathrm{T}$ cell and its mechanism of suppression. Nat. Med., 6: 782-789.

Zhao, A.G., H.L. Zhao, X.J. Jin, J.K. Yang and L.D. Tang, 2002. Effects of Chinese Jianpi herbs on cell apoptosis and related gene expression in human gastric cancer grafted onto nude mice. World J. Gastroenterol., 8: 792-796.

Zhao, N., W. Zhang, Y. Guo, H. Jia and Q. Zha et al., 2010. Effects on neuroendocrinoimmune network of Lizhong Pill in the reserpine induced rats with spleen deficiency in traditional Chinese medicine. J. Ethnopharmacol., 133: 454-459.

Zheng, J., Z. Qu, C. Luo, Z. Hu, J. Li and Y. Wei, 2007. The electrogastrogram in cows and goats with $\mathrm{Pi}-\mathrm{xu}-$ zheng (spleen deficiency pattern). Am. J. Traditional Chin. Vet. Med., 2: 12-22.

Zhou, X., S. Chen, B. Liu, R. Zhang and Y. Wang et al., 2010. Development of traditional Chinese medicine clinical data warehouse for medical knowledge discovery and decision support. Artificial Intell. Med., 48: 139-152. 